\title{
Nonresonant Control of Multimode Molecular Wave Packets at Room Temperature
}

\author{
R. A. Bartels, * T. C. Weinacht, S. R. Leone, H. C. Kapteyn, and M. M. Murnane \\ JILA, University of Colorado and National Institutes of Technology, Boulder, Colorado 80309
}

(Received 1 June 2001; published 4 January 2002)

\begin{abstract}
We demonstrate the creation and measurement of shaped multimode vibrational wave packets with overtone and combination mode excitation in $\mathrm{CCl}_{4}$. Excitation of wave packets through nonresonant impulsive stimulated Raman scattering allows for coherent control of molecular vibrations without passing through an electronic resonance. This technique is therefore very general and can be implemented in a large class of molecular gases and liquids at STP, which were previously inaccessible because their resonances are in the VUV.
\end{abstract}

DOI: $10.1103 /$ PhysRevLett.88.033001

With the advent of ultrafast lasers capable of generating pulses that are short in comparison to molecular time scales, there has been considerable interest in creating and measuring vibrational wave packets. Early experiments generated wave packets in excited electronic states and demonstrated control over the position of these wave packets as a function of time [1]. Recent experiments [2] have included nuclear motion in the ground electronic state, which is directly relevant to controlling and monitoring many chemical reactions. These experiments focused on controlling motion in a single normal mode of the system. Extending control and measurement of vibrational motion to several modes including overtones is an exciting prospect for several types of studies, including mode coupling and bond selective chemistry [3,4]. Furthermore, most past work on ground state vibrations relied on transferring population through an excited electronic state (resonant Raman). In this Letter we demonstrate the creation and measurement of shaped multimode vibrational wave packets in $\mathrm{CCl}_{4}$ with strong overtone excitation, implying a significant vibrational amplitude. We also control which modes and which mode combinations are excited. A learning algorithm is used to automate the control process. Past work in $\mathrm{CCl}_{4}$ used sequences of five pulses to excite vibrational modes to investigate intramode coupling and anharmonicity, although no overtone or combination modes were observed [5].

The vibrational wave packets in this work are excited with a single pulse far detuned from electronic resonances, achieving nonresonant control of the amplitude of modes in the wave packet, as shown in earlier solid state impulsive stimulated Raman scattering (ISRS) experiments $[6,7]$. Because it is nonresonant, it can be generalized to any molecular gas or liquid with Raman-active modes that can be excited impulsively, and it is not limited by the need to access resonances in the VUV. This approach works at high pressures and temperatures, and thus may facilitate laser control of chemical reactions with macroscopic product yields. In particular, since changes in chemical bonding will involve a superposition of several modes, control of multimode wave packets may lead to selective control over reactions.
PACS numbers: 33.80.-b, 42.65.Dr

Excitation of Raman-active modes involves two photons with a frequency difference equal to the vibrational energy. If the duration of a driving laser pulse $\tau_{p}$ is shorter than the vibrational period $\tau_{\nu}=2 \pi / \Omega_{\nu}$, the bandwidth of the laser pulse $\Delta \omega_{L}$ exceeds the vibrational frequency, and the excitation can be considered impulsive. During ISRS, frequency pairs with a difference of $\Omega_{\nu}$ within the bandwidth of a single laser pulse act as pump and Stokes photons. For appropriate phasing of pairs within the bandwidth, strong excitation results. In a simple classical model, the displacement from equilibrium of the nuclear coordinate $Q$ for a given mode $\nu$ in the ISRS limit can be described by [8] $\frac{d Q^{2}}{d t^{2}}+2 \gamma \frac{d Q}{d t}+\Omega_{\nu}^{2} Q=\frac{1}{2} N\left(\frac{d \alpha}{d Q}\right)_{0} E_{L}^{2}$, where $\gamma$ is the coherence decay rate, $Q_{\nu}$ is the vibrational frequency, $N$ is the number density of molecules, $(d \alpha / d Q)_{0}$ is the change in polarizability with intermolecular distance $\mathrm{Q}$, and $E_{L}(t)=\frac{1}{2}\left[A_{L}(t) e^{-i \omega_{L} t}+\right.$ c.c. $]$ is the laser field. Expanding the laser field as $E_{L}^{2}(t)=\frac{1}{4}\left\{2 A_{L}(t) A_{L}^{*}(t)+\right.$ $\left.A_{L}^{2} e^{-i 2 \omega_{L} t}+A_{L}^{* 2} e^{i 2 \omega_{L} t}\right\}$ gives a "dc" term corresponding to the intensity profile $I(t)$, and two second harmonic terms. The nuclei, which have too much inertia to respond to the fundamental (or second harmonic) field, are effectively driven by $I(t)$. Thus, the strength of excitation of a vibrational mode with frequency $\Omega$ is proportional to the power spectral density $D(\Omega)$ of the intensity profile at that vibrational frequency, where $D(\Omega)=\int_{-\infty}^{\infty} d \omega \times$ $a_{L}(\omega) a_{L}^{*}(\Omega-\omega) \exp \{-i[\phi(\omega)-\phi(\Omega-\omega)]\} \quad$ and $A(\omega)=\mathcal{F}\{A(t)\}=a_{L}(\omega) \exp [-i \phi(\omega)]$. Based on this simple analysis, a pulse with spectral phase described by a periodic function at the molecular vibrational frequency (e.g., a train of pulses separated by the vibrational period $[6,8]$ ) should be as efficient as a transform-limited pulse in exciting vibrations $[6,8,9]$. [Note: to maximize $D(\Omega)$, $\phi(\omega)-\phi(\Omega-\omega)=$ const.] Later in the text, we compare the excitation of Raman modes with intense transformlimited pulses to that of low-intensity chirped Raman excitation and observe the breakdown of the weak excitation limit described by this analysis.

For our experiments, we used $15 \mathrm{fs}\left(\sim 660 \mathrm{~cm}^{-1}\right.$ FWHM bandwidth) laser pulses generated by an amplified Ti:sapphire laser system, at a $1 \mathrm{kHz}$ repetition frequency, and with an energy of $\sim 1 \mathrm{~mJ}[10]$. The pulses from our laser 
were shaped using two separate techniques. The laser is equipped with a deformable mirror pulse shaper, which alters the spectral phase of the output pulses. Alternatively, a pulse pair generated by a Michelson interferometer can be used for somewhat less flexible control. This pulse pair can be either transform limited or "chirped" by adjusting a pair of gratings in the pulse compressor. By adjusting the relative delay of the chirped or compressed pulse pair, we control which modes in the wave packet are excited. A small portion of the pump pulse $(\sim 10 \%)$ is used as a probe of the Raman coherence. This probe pulse is frequency doubled in a long nonlinear crystal to generate a narrow bandwidth (narrower than the separation between modes in $\left.\mathrm{CCl}_{4}\right)$ probe $(\approx 1 \mathrm{THz}, 450 \mathrm{fs}, 400 \mathrm{~nm})$ that is well separated spectrally from the pump.

The pump and probe pulses are focused into a $30-\mathrm{cm}-$ long, 450- $\mu \mathrm{m}$-diameter hollow-core fiber filled with a molecular gas or vapor from a molecular liquid at pressures of 100-900 torr [11,12]. The pump pulse excites a vibrational coherence in the molecules, which is detected by the probe as a time-varying index of refraction. This results in the appearance of FM sidebands on the probe spectrum, separated by the vibrational frequency. Monitoring the intensity of a given sideband provides a measure of the vibrational excitation of the mode labeled by the sideband.

Figure 1 shows the results of selective excitation of vibrational modes in $\mathrm{CCl}_{4}$ using impulsive chirped Raman excitation. For this experiment, the pulse was first chirped in time (to $\sim 83 \mathrm{fs}$, with a chirp rate of $0.1125 \mathrm{THz} / \mathrm{fs}$ ) prior to entering the Michelson interferometer. The instantaneous frequency $\left[\omega_{i}=d \varphi(t) / d t=2 b \tau\right.$, where $c_{R}=b / \pi$ is the chirp rate as defined by $\left.\varphi(t)=\omega_{L} t+b t^{2}\right]$ of the pulse sweeps linearly in time, producing a constant frequency difference $(\Delta \nu)$ between the two pulses from the interferometer for nonzero delays. The interference between the two pulses produces a beat note with a period of $1 / \Delta \nu$ in the intensity profile described by $I_{t}(t)=$ $I_{\text {in }}(t)+I_{\text {in }}(t-\tau)+2 \sqrt{I_{\text {in }}(t) I_{\text {in }}(t-\tau)} \cos \left(\omega_{L} \tau+b \tau^{2}-\right.$ $2 b \tau t)$, where $I(t)$ is the intensity profile, $\tau$ is the delay between the pulses, and $\Delta \nu=c_{R} \tau$. Changing the delay tunes the frequency difference and the period of the intensity modulations, allowing for mode selective excitation of the molecule. The chirped pulses were injected into the hollow-core fiber filled with a room-temperature vapor pressure ( $\sim 80$ torr) of $\mathrm{CCl}_{4}$. The three Raman modes under control are $\nu_{1}, \nu_{2}$, and $\nu_{4}$, whose frequencies are $13.77\left(459 \mathrm{~cm}^{-1}\right), 6.54\left(218 \mathrm{~cm}^{-1}\right)$, and $9.42\left(314 \mathrm{~cm}^{-1}\right)$ $\mathrm{THz}$, respectively. Figure 1(a) shows the probe spectrum as a function of interferometer delay. As the frequency difference in the pump matches the vibrational frequency of each mode in the molecule, that mode is driven efficiently while the other modes remain relatively unaffected.

Figure 1(b) shows lineouts of the scattered probe intensity at frequencies corresponding to each of the fundamental modes. For a fixed chirp rate (pulse length), the optimal

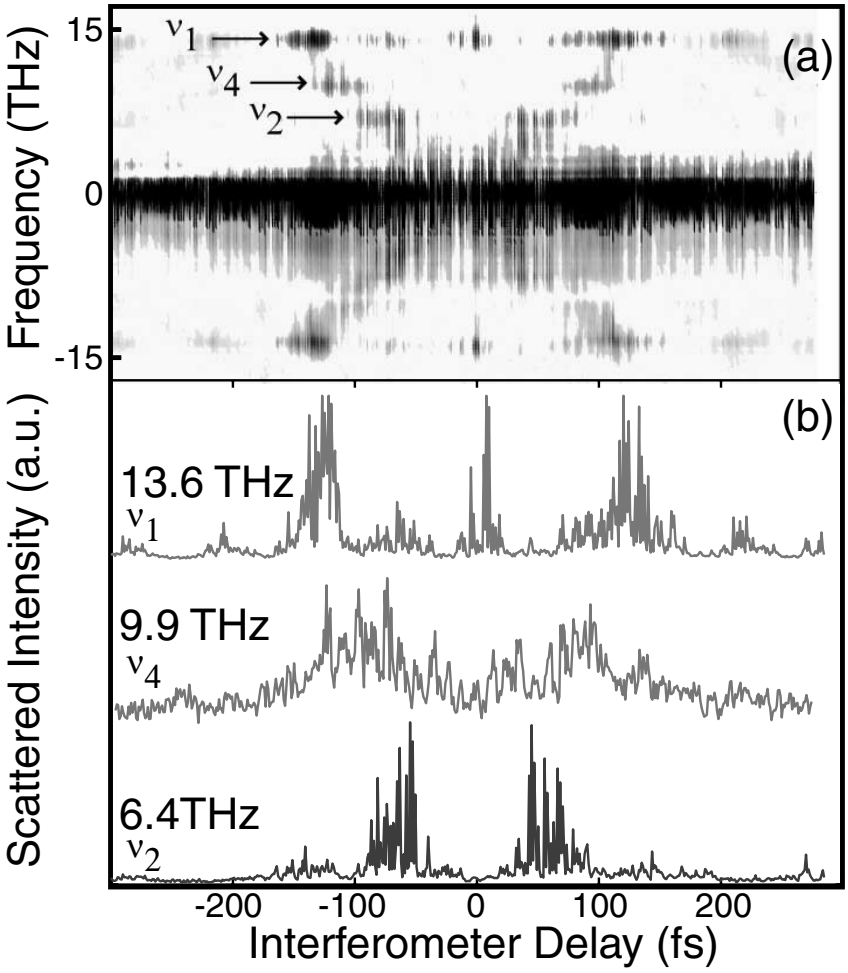

FIG. 1. Probe spectrum of $\mathrm{CCl}_{4}$ when excited by two chirped pulses. The shading in the figure is proportional to the scattered light intensity. The chirped pulse is still sufficiently short to drive some ISRS excitation near time zero. (a) Probe spectrum vs delay between pump pulses. (b) Lineouts at vibrational frequencies $\nu_{1}, \nu_{4}$, and $\nu_{2}$.

delay for mode $\nu_{1}$ is given by $\tau_{1}=\Omega_{1} / c_{R}$. The optimal time delays for modes $\nu_{1}, \nu_{4}$, and $\nu_{2}$ appear at 122, 88, and $58 \mathrm{fs}$, implying frequencies of 13.6, 9.9, and $6.5 \mathrm{THz}$, respectively. This technique also works well in other molecules such as $\mathrm{SF}_{6}[12]$. The data show that vibrational modes within the laser bandwidth can be separately controlled with a properly constructed $I(t)$.

Excitation of Raman modes in $\mathrm{CCl}_{4}$ exhibits different behavior when driven by intense, transform-limited pulses. Figure 2 shows the probe spectrum obtained after excitation of $\mathrm{CCl}_{4}$ with two time-delayed transform-limited pulses. Excitation with transform-limited pulses can lead to overtone excitation, as shown in Fig. 3(b). This can also be seen by performing a 1D Fourier transform of the data in Fig. 2(a) to produce a data set that can be compared with that of Fig. 1(a). Figure 3(a) shows such a transform in which various modes and mode combinations can be seen. The two transform-limited pulses excite not only the normal modes of the system but also overtones and combination modes not observed in the chirped pulse data, as shown in Figs. 2 and 3.

The excitation mechanism is best understood in complementary domains for the two experiments. For transformlimited pulses, the first pulse creates a wave packet that begins to evolve. The second pulse then generates another 


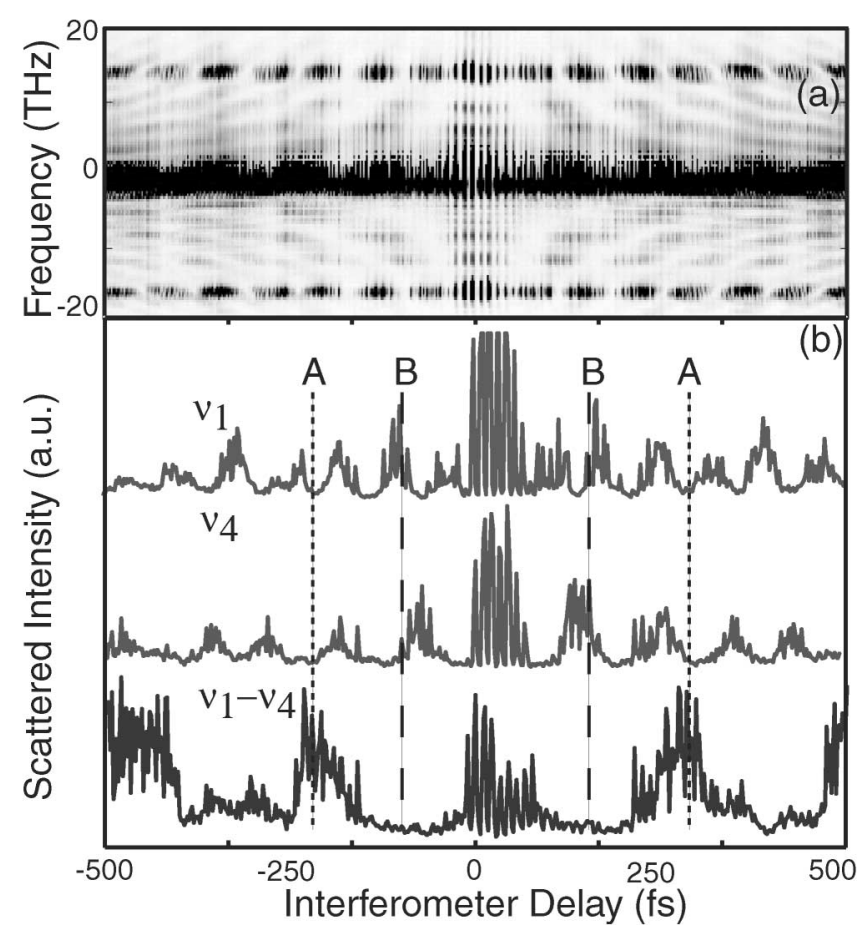

FIG. 2. Probe spectrum of $\mathrm{CCl}_{4}$ excited by two transformlimited pulses. (a) Probe spectrum vs time delay between pump pulses. (b) Lineouts at frequencies corresponding to $\nu_{1}, \nu_{4}$, and $\nu_{1}-\nu_{4}$.

wave packet, which interferes with the first, enhancing or suppressing modes in the wave packet depending on the delay between pulses. The case of chirped excitation pulses is best understood in the frequency domain. A chirped broadband pulse can be thought of as a comb of $\sim 10^{9}$ phase-locked quasi-cw lasers spread out in time (reducing the peak intensity by spreading energy in time), tuning from red to blue. The second time-delayed pulse causes interference between two spectrally shifted combs, equivalent to detuning a $\mathrm{cw}$ laser by an amount proportional to the delay time and the chirp rate. For a purely linear response, we expect the same behavior for both cases. The differences we observe indicate a nonlinear response of the molecular system to an intense transform-limited pulse.

The light scattered at the frequency $2 \nu_{1}$ in Fig. 3(a) shows two local maxima, only one of which lies on the line of slope 1 . The maximum occurring with a pulse periodicity of $1 / \nu_{1}$ cannot be unambiguously assigned to light scatter from the overtone as it may include light scattered twice from $\nu_{1}$. The second spot marked is scattered to a frequency $2 \nu_{1}$ and has a periodicity of $\sim 1 / 2 \nu_{1}$, providing unambiguous evidence for overtone excitation. Further evidence for overtone excitation is revealed by observing the dephasing and rephasing of the wave packets due to the anharmonic potential. Figure 3(b) shows the results of sweeping the probe delay while monitoring light scattered to $13.77 \mathrm{THz}$ in $\mathrm{CCl}_{4}$. A $10.6 \pm 0.7 \mathrm{ps}$ beat period is observed, corresponding to an anharmonicity $\left(2 \omega_{e} x_{e}\right)$ of $0.0948 \pm 0.006 \mathrm{THz}\left(3.16 \pm 0.2 \mathrm{~cm}^{-1}\right)$.
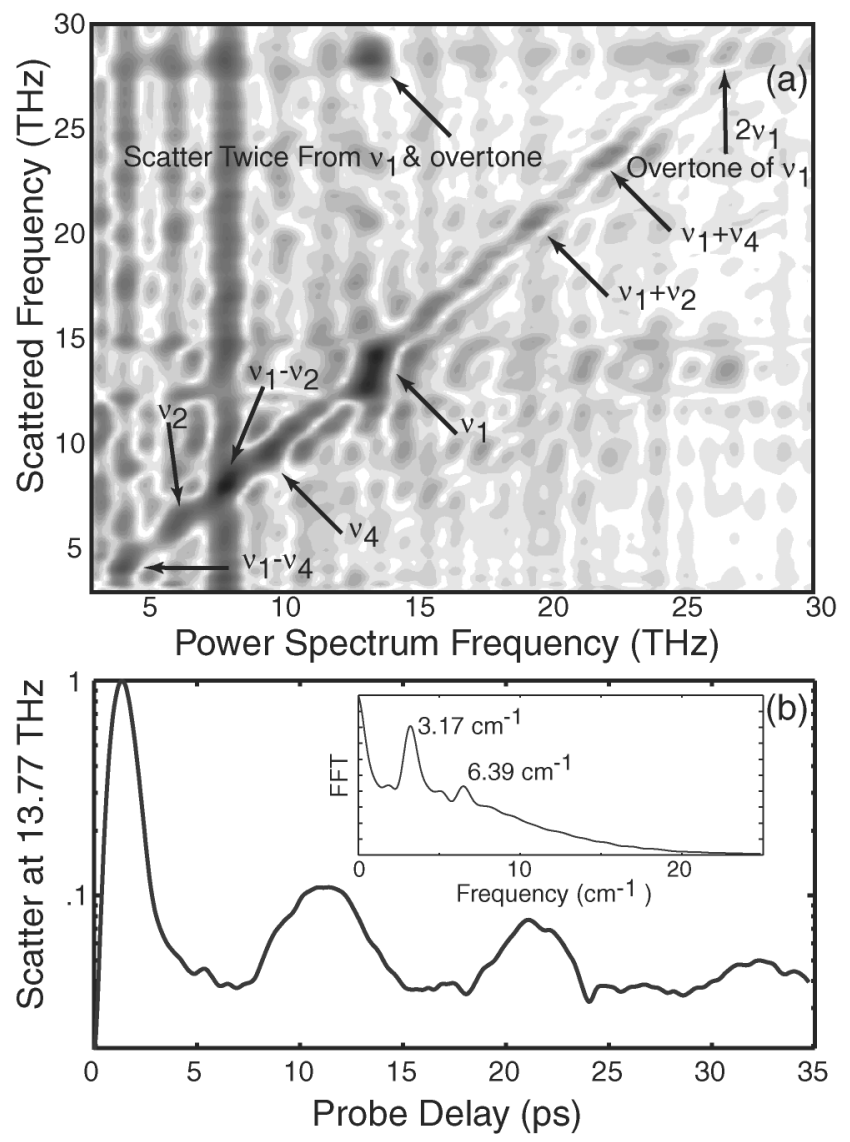

FIG. 3. (a) Data from Fig. 2, where the temporal data at each scattered frequency is Fourier transformed. The shaded regions indicate the power spectral density. (b) Scattered probe light at 13.77 $\mathrm{THz}$ in $\mathrm{CCl}_{4}$ shows beating due to overtone excitation. The fast-Fourier transform (FFT) of the time delay scan in the inset shows excitation in both the first and second overtones.

Fourier transforming the time scan reveals evidence for higher overtone excitation [Fig. 3(b) inset]. The depth of modulation, due to the level of overtone excitation, is seen to decay faster than the fundamental vibration coherence (estimated to be $18 \mathrm{ps}$ ). Detailed knowledge of the vibrational potential should allow for determination of the eigenstate populations [13]. We also observe evidence for overtone excitation in $\mathrm{CO}_{2}$ for the asymmetric stretch mode $\nu_{1}(41.64 \mathrm{THz})$. We measure rephasing with a period of $1.47 \pm 0.1 \mathrm{ps}$, implying a $0.681 \pm 0.093 \mathrm{THz}$ $\left(22.7 \pm 3.1 \mathrm{~cm}^{-1}\right)$ anharmonicity, in agreement with published values [14]. An alternate technique can measure the anharmonicity of IR active modes in organic molecules by observing the beating of vibrational echo signals initiated by pairs of IR free electron laser pulses [15]. In contrast, our technique measures the anharmonicity of Raman active modes with nonresonant ISRS excitation.

The lineouts of Fig. 2(b) demonstrate our ability to excite a combination mode that is a coherent sum of two different normal modes. By adjusting the delay between pump pulses to $1 /\left(\nu_{1}-\nu_{4}\right)$, we can excite vibrational motion that is a combination of the $\nu_{1}$ and $\nu_{4}$ modes of the 


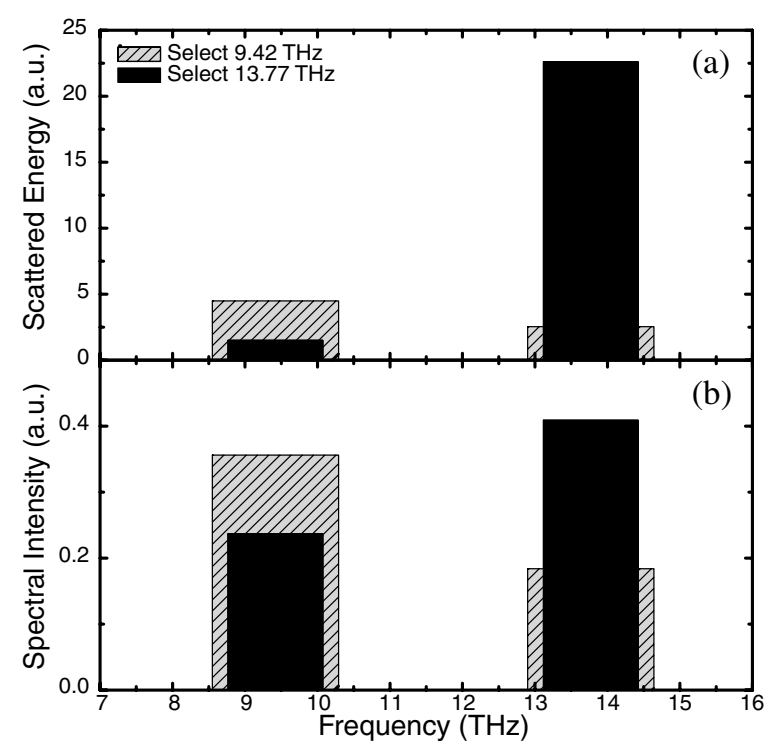

FIG. 4. Learning control and selective excitation of two Ramanactive modes in $\mathrm{CCl}_{4}$. (a) Enhancement of either the $13.77 \mathrm{THz}$ mode (black, optimizing $\nu_{1}$ vs $\nu_{4}$ ) or the $9.42 \mathrm{THz}$ mode (hatched, optimizing $\nu_{4}$ vs $\nu_{1}$ ). (b) Power spectrum of the optimized pulse intensity profile.

molecule [lowest lineout in Fig. 2(b), at position $A$ ], where no individual excitation of modes $\nu_{1}$ or $\nu_{4}$ is observed. This indicates that the probe does not simply acquire modulations at the difference frequency through optical mixing of the two normal mode frequencies, but rather that it is modulated by the motion of the molecule in the combination mode. Position $B$ marks a delay between pump pulses for which the excitation of both normal modes is maximum, but where there is no excitation of the combination mode. Excitation of such combination modes is an important step toward bond selective excitation, since reaction coordinates do not always correspond to normal modes of molecules.

Finally, we also demonstrate an alternate pulse shaping scheme based on a deformable mirror pulse shaper [16], together with a feedback loop [17] that incorporates a learning algorithm [18]. This approach allows for more general shaping of a multimode wave packet because the pulse shaper is programmable and can be guided by the learning algorithm. For example, exciting $\mathrm{CCl}_{4}$ with a single, short pulse coherently excites all three Raman-active vibrational modes $\left(\nu_{1}, \nu_{2}, \nu_{4}\right)$ simultaneously. Tailoring the intensity profile allows us to control the excitation of each vibrational mode independently. In this experiment, an evolutionary algorithm was used to direct the pulse shaper to excite a specific mode $\left(\nu_{1}\right)$ while suppressing others by adjusting the relative spectral phases in the pulse. The black bar in Fig. 4(a) shows that the relative energy scattered into $\nu_{1}$ is over 10 times larger than for $\nu_{4}$. The same trend is reflected in the power spectrum of the intensity profile at the vibrational frequencies [Fig. 4(b)]. The reverse experiment (optimizing $\nu_{4}$ vs $\nu_{1}$ ) was also performed. The degree of control shown here is limited by the resolution of the pulse shaper. Although an algorithm is not, in principle, required for this technique, it is very convenient because it compensates for any experimental nonidealities and automates the tailoring of populations (mode amplitudes) in the wave packet by selecting which modes participate.

In conclusion, we have demonstrated the ability to create "custom" multimode vibrational wave packets in molecular systems at STP. Significant vibrational amplitude is achieved as evidenced by strong overtone excitation. In addition to creating shaped molecular wave packets, we measure and observe dynamics using temporally resolved nonlinear spectroscopy. These techniques are general and can be applied to many transparent molecular gases, or liquids with high vapor pressures at room temperature.

We acknowledge useful discussions with David Nesbitt, David Jonas, and Paul Corkum. The authors gratefully acknowledge support for this work from the National Science Foundation.

*Author to whom correspondence should be addressed. Email address: bartels@colorado.edu

[1] B. Kohler et al., Phys. Rev. Lett. 74, 3360 (1995).

[2] G. Knopp et al., J. Raman Spectrosc. 31, 51 (2000); V. Lozovoy et al., ibid.31, 41 (2000); T. Hornung et al., Chem. Phys. Lett. 326, 445 (2000); M. Karavitis et al., J. Chem. Phys. 114, 4131 (2001); E. Hertz et al., Phys. Rev. A 61, 033816 (2000).

[3] F. Crim, Acc. Chem. Res. 32, 877 (1999).

[4] R. Zare, Science 279, 1875 (1998).

[5] A. Tokmakoff et al., Phys. Rev. Lett. 79, 2702 (1997).

[6] L. Dhar, J. Rogers, and K. A. Nelson, Chem. Rev. 94, 157 (1994).

[7] A. M. Weiner et al., Science 247, 1317 (1990).

[8] Y.X. Yan, E. B. Gamble, Jr., and K. A. Nelson, J. Chem. Phys. 83, 5391 (1985).

[9] D. Meshulach and Y. Silberberg, Phys. Rev. A 60, 1287 (1999).

[10] E. Zeek et al., Opt. Lett. 25, 587 (2000).

[11] A. Nazarkin et al., Phys. Rev. Lett. 83, 2560 (1999).

[12] T. Weinacht et al., Chem. Phys. Lett. 344, 333 (2001).

[13] V. Romero-Rochín et al., J. Chem. Phys. 111, 3559 (1999).

[14] Q. Meng, Y. Zheng, and S. Ding, Int. J. Quantum Chem. 81, 154 (2001).

[15] K. D. Rector et al., J. Chem. Phys. 106, 10027 (1997).

[16] E. Zeek et al., Opt. Lett. 24, 493 (1999).

[17] R. Bartels et al., Nature (London) 406, 164 (2000).

[18] R. S. Judson and H. Rabitz, Phys. Rev. Lett. 68, 1500 (1992). 